Provided for non-commercial research and education use. Not for reproduction, distribution or commercial use.

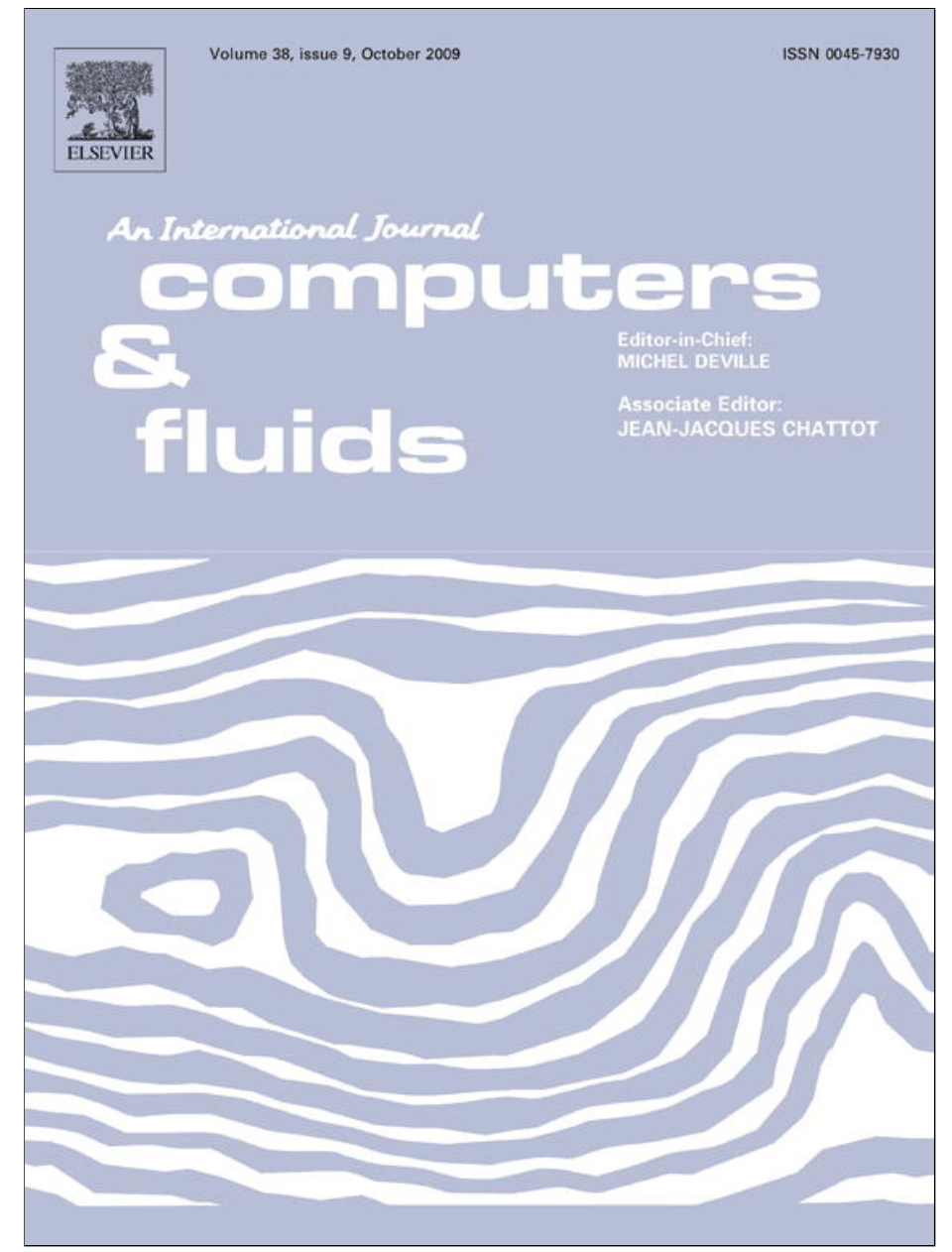

This article appeared in a journal published by Elsevier. The attached copy is furnished to the author for internal non-commercial research and education use, including for instruction at the authors institution and sharing with colleagues.

Other uses, including reproduction and distribution, or selling or licensing copies, or posting to personal, institutional or third party websites are prohibited.

In most cases authors are permitted to post their version of the article (e.g. in Word or Tex form) to their personal website or institutional repository. Authors requiring further information regarding Elsevier's archiving and manuscript policies are encouraged to visit:

http://www.elsevier.com/copyright 


\title{
Domain decomposition for near-wall turbulent flows
}

\author{
S.V. Utyuzhnikov* \\ School of Mechanical, Aerospace E Civil Engineering, University of Manchester, Sackville Street, P.O. Box 88, Manchester M60 1QD, UK
}

\section{A R T I C L E I N F O}

\section{Article history:}

Received 20 October 2008

Received in revised form 27 February 2009

Accepted 9 March 2009

Available online 20 March 2009

\begin{abstract}
A B S T R A C T
Modeling near-wall high-Reynolds-number turbulent flows is a time-consuming problem. A domain decomposition approach is developed to overcome the problem. The original computational domain is split into a near-wall (inner) subdomain and an outer subdomain. The developed approach is applied to a model 2D equation simulating major peculiarities of near-wall high-Reynolds-number flows. On the base of the Calderon-Ryaben'kii potential theory it is possible to consider the near-wall (inner) problem independently on the outer problem. The influence of the inner problem can exactly be represented by a pseudo-differential equation formulated on the intermediate boundary. In a 1D case, it leads to the wall functions represented by Robin boundary conditions, which can be determined either analytically or numerically. It is important that the wall functions (or boundary conditions) are mesh independent and can be realized in a separate routine. Thus, the original problem can only be solved in the outer domain with some specific nonlocal boundary conditions called nonlocal wall functions. The technique can be extended to 3D problems straightforward.
\end{abstract}

(c) 2009 Elsevier Ltd. All rights reserved.

\section{Introduction}

It is well known that near-wall turbulent flows are very complicated for numerical simulation. Due to the no-slip boundary condition for the velocity and wall damping effect, a so called laminar sub-layer appears in the vicinity of the wall. Although the laminar sub-layer is very thin, its resolution strongly affects the accuracy of the solution in the entire domain. Thereby, high-Reynolds-number near-wall flows are characterized by profiles with high gradients and their resolution requires a fine mesh nearby the wall. The influence of the wall leads to strong flow anisotropy. Inevitably, the Boussinesq hypothesis, used in many models of the Reynoldsaveraged-Navier-Stokes equations (RANS), fails. This demands the development of so called low-Reynolds-number models which include complicated semi-empirical near-wall correction terms. Practical computations of industrial flows showed that accurate enough near-wall resolution takes, at least, half the total computational time [1]. The use of Large Eddy Simulation (LES) technique allows one to avoid complicated semi-empirical near-wall models. However, this approach is very time-consuming because the near-wall vortexes are determined by the distance to the wall and become very small. Thereby, correct near-wall resolution is very problematic and often not realistic in industrial computations. Hybrid approaches [2] based on the use of domain decomposition with RANS models nearby the wall and LES outside seem more efficient, however the problem of the near-wall resolution retains.

\footnotetext{
* Tel.: +44 161306 3707; fax: +44 1613063723 .

E-mail address: s.utyuzhnikov@manchester.ac.uk
}

The high-Reynolds-number model also uses the decomposition approach. In this model the RANS equations are applied in the main flow, while the near-wall domain is not numerically resolved. Instead, some boundary conditions called wall functions are set on some boundary nearby the wall usually assigned with the first near-wall cell. The wall functions are based on a local approximation of the near-wall flow. First wall functions [3], suggested by Launder and Spalding, corresponded to the analytical solution of the classical problem on a thin plane and were based on the logprofile. Although such an approximation is quite acceptable for relatively simple near-wall flows, it is not good enough for complex geometries and flows with strong source terms such as the pressure gradient and buoyancy forces. Nowadays, different modifications of the wall functions have been suggested. Most of them use some empirical parameters. If the boundary conditions (wall functions) appear accurate enough, the high-Reynolds-number models are very efficient for practical applications. The problem is that we are often not able to estimate the applicability of the wall functions until we know the solution to be found. Approaches based on an approximate near-wall integration allow one to obtain more universal wall functions [4-6]. Another significant problem related to most wall functions is a high sensitivity of the solution to the size of the near-wall cells where the wall functions are set [1,7-9].

In [10], wall functions are interpreted as the boundary conditions transferred from the wall to some boundary nearby the wall. The approach has first been developed for a 1D model equation. It appears that such wall functions are mesh independent, represented by Robin boundary conditions, and take into account source terms. Later, the approach has been applied to the RANS equations 
with the $k-\epsilon$ model. The near-wall governing equations are represented by the Reduced Navier-Stokes equations [11] with "frozen" derivatives along the wall. The wall functions are derived for all variables but $\epsilon$ in a uniform manner and do not contain any free parameters. The Robin-type wall functions have been applied for the study of channel flow and impinging jet at very different input parameters in $[12,13]$. The computations confirmed a reasonably weak dependence of the solution on the near-wall mesh distribution. In [14], it is shown that the Robin-type wall functions can be obtained via the theory of the Calderón-Ryaben'kii potentials $[15,16]$. This theory allows us to reduce a boundary value problem (BVP) in a quite arbitrary domain to a boundary equation without the use of Green's function. The boundary equation is represented by a pseudo-differential equation. In [14], this technique is locally used in the normal to the wall direction.

In the current paper, the approach, derived in [14] for a 1D local equation, is extended to a multidimensional case. For this purpose, a model $2 \mathrm{D}$ equation is considered. The equation represents a generalization of the model equation used in [10]. Nearby the wall the solution of the model equation contains a boundary-layer profile. Thereby, the flow field can be split into high-gradient flow nearby the wall and an external field. Using the technique based on the Calderón-Ryaben'kii potential theory [15,16], the boundary conditions are transferred from the wall to some intermediate boundary which separate high-gradient inner and outer domains. It is very important to know that the boundary conditions can be transferred in advance without the consideration of the outer domain. Thereby, they can be applied to different external boundary conditions and different geometries. The boundary conditions on the intermediate boundary, represented by a pseudo-differential equation, are nonlocal. If the boundary conditions are exactly transferred from the wall, we obtain a decomposition method. Alternatively, the boundary conditions can be transferred approximately. This leads to a multidimensional generalization of the Robin-type wall functions. In other words, for the first time we obtain nonlocal wall functions. The nonlocal wall functions capture main flow features along the wall such as high longitudinal derivatives caused by either source terms or the curvature of the wall. Although the approach is applied to a $2 \mathrm{D}$ equation, it can naturally be realized in a 3D case.

The paper is organized as follows: in Section 2, the general formulation of a model BVP and domain decomposition are introduced. Then, in Section 3, the boundary conditions are transferred from the real boundary to an intermediate boundary via the CalderónRyaben'kii potentials. This technique leads to some kind of the $D-$ $t-N$ map. First, the partial case of a positive definite operator is considered in Section 4 . The $D-t-N$ map is obtained in the explicit analytical form. Then, the approach is generalized to the operator of a general kind. The $D-t-N$ map can be realized numerically as shown in Section 5 The concept of the nonlocal wall functions is also introduced there. The numerical example of the use of nonlocal functions and domain decomposition is given in Section 8.

\section{Model equation and domain decomposition}

Let us consider the following BVP formulated on cylinder $D:=\Omega \times[01]$ :

$L U=f(\mathbf{x}, y)$,

$l_{y} U(\mathbf{x}, 0)=\alpha_{w}(\mathbf{x})$

$l_{\mathbf{x}} U_{\mid \gamma_{c}}=\beta_{w}(y)$

$U(\mathbf{x}, 1)=U_{s}(\mathbf{x})$.

Here, $\mathbf{x} \in \Omega \subset \mathbb{R}^{m}, m=1,2 ; \Omega$ is a single-connected domain with piece-wise smooth boundary $\partial \Omega ; \gamma_{c}:=\partial \Omega \times[01]$. Assume that $L$ is a second-order linear differential operator; $l_{\mathbf{x}}$ and $l_{y}$ are first-or- der linear differential operators at the boundary with respect to $\mathbf{x}$ and $y$. We suppose that BVP (1) and (2) is well-posed according to Hadamard.

As a partial case of BVP (1), we further consider the following problem on square $\left[\begin{array}{ll}0 & 1\end{array}\right] \times\left[\begin{array}{ll}0 & 1\end{array}\right]$ :

$\left(\mu(y) U_{y}\right)_{y}+\sigma(y) U_{x x}+\beta(y) U_{y}+\gamma(y) U=f(x, y)$,

$l_{y} U(x, 0)=\alpha_{w}(x)$,

$U(0, y)=U_{0}(y)$

$U(1, y)=U_{1}(y)$

$U(x, 1)=U_{s}(x)$.

where $\sigma=\alpha \mu, \alpha>0, \mu=(1-\exp (-y / \epsilon)+\delta) / R e, \epsilon \ll 1, \delta \ll 1$, $\operatorname{Re} \gg 1, \beta=C y^{p}, p>0$.

In the governing equation (3), the first two terms on the left-hand side simulate dissipative terms in the RANS equations, whereas the other terms model convective terms, and the right-hand side corresponds to the source terms such as the pressure gradient. The coefficient $\mu$ represents the effective viscosity coefficient. It is to be noted that a partial case of the model equation (3) has been considered in [10]. The equation, used in [10], was the model equation for the Robin-type 1D wall functions [13]. Later [17], this equation was used for the development of wall-functions in LES.

It is clear that the parameter $\alpha$ in BVP (3)-(5) is responsible for the nonlocal effects in $x$-direction. A typical example of such solutions is flow around a corner with a high curvature. If the coordinate $x$ is related to the wall, the coefficient $\alpha$ can be proportional to the curvature squared.

Next, we consider a domain decomposition: $D:=D_{1} \cup D_{2}$, where $D_{1}:=\Omega \times\left[0 y^{*}\right], 0<y^{*}<1$ and $D_{2}:=D \backslash \bar{D}_{1}$. We intend to split BVP (1) into two BVPs formulated on the domains $D_{1}$ and $D_{2}$. According to the physical sense of the introduced model BVP, we assume that a BVP in the "near-wall" domain $D_{1}$ corresponds to a high-gradient solution with predominant gradients in the $y$-direction. Our task is to separate the solution in the domain $D_{1}$ from the solution in the rest domain. Such a separation is not trivial because the boundary conditions at the intermediate boundary $y=y^{*}$ are unknown. It appears that in the linear case, at least, the boundary condition at $y=0$ can exactly be transferred to the intermediate boundary $y=y^{*}$. However, this boundary condition is not local any more. In a $1 \mathrm{D}$ case, this approach is completed in $[10,14]$.

The domain decomposition can efficiently be realized via the theory of potentials. In the next Section, a brief introduction to the theory of the Calderón-Ryaben'kii potentials in application to the problem in question is given. It is shown that there is an immediate relation between this theory and the domain decomposition to be done. A detailed information on the theory of Calderón-Ryaben'kii potentials can be found in $[15,16]$.

\section{Domain decomposition and the Calderón-Ryaben'kii potentials}

Let us denote the space of smooth enough functions satisfying boundary conditions (2) by $\Xi_{D}$. In addition, we require that the solution of Eq. (1) is unique for any right-hand side $f: f=$ $L V, V \in \Xi_{D}$.

Strictly speaking, under the solution of BVP (1) and (2) we understand a generalized solution: $\langle L U, \Phi>=<f, \Phi>$ for any test function $\Phi \in C_{0}^{\infty}(D)$. Here, $<f, \Phi>$ denotes a linear functional associated with a given generalized function $f, C_{0}^{\infty}(D)$ is the space of infinitely differentiable functions vanishing at the boundary of $D$. Details can be found in [16].

Then, we introduce a potential in the domain $D_{1}$ as follows [15]:

$P_{D_{1}} V_{D_{1}}(\mathbf{z}) \stackrel{\text { def }}{=} V_{D_{1}}-\int_{D_{1}} G_{D}(\mathbf{z}, \mathbf{y}) L V(\mathbf{y}) d \mathbf{y}, \quad \mathbf{z}, \mathbf{y} \in \mathbb{R}^{m+1}$. 
Here and further, $V_{D_{1}}$ is the restriction of a function $V \in \Xi_{D}$ to $D_{1} ; G_{D}$ is the Green's function of BVP (1) and (2) with $f=L V$. It should be noted that, in fact, explicit Green's function is not necessarily required. In practical applications, it can efficiently be replaced by Green's operator [15].

It appears that the potential does not depend on the complementation of $V_{D_{1}}$ to any function $V^{\prime} \in \Xi_{D}[15,16]$. In addition, one can prove [15] that the potential satisfies the homogeneous equation on the domain $D_{1}$ :

$L P_{D_{1}} V_{D_{1} \mid D_{1}}=0_{D_{1}}$

Next, let $\Gamma$ be the boundary of $D_{1}: \Gamma:=\partial D_{1}$. Following [15], let us introduce the operator of a clear trace $\operatorname{Tr}_{\Gamma}: \Xi_{D} \rightarrow \Xi_{\Gamma}$ and

$\operatorname{Tr}_{\Gamma} V_{D_{1}}=\operatorname{Tr}_{\Gamma} W_{D_{1}} \Rightarrow P_{D_{1}} V_{D_{1}}=P_{D_{1}} W_{D_{1}}$,

if $V \in \Xi_{D}, W \in \Xi_{D}$. Here, $\Xi_{\Gamma}$ is the appropriate space of functions determined on the boundary $\Gamma[16]$.

Thus, the potential is fully determined by the clear trace, and one can reduce the volume potential to a surface potential with density $\xi_{\Gamma}$ :

$P_{D_{1} \Gamma} \xi_{\Gamma} \stackrel{\text { def }}{=} P_{D_{1}} V_{D_{1}}$

where $\xi_{\Gamma}=\operatorname{Tr}_{\Gamma} V_{D_{1}}$

One can prove that the clear trace $\xi_{\Gamma}$ of any function $V \in \Xi_{D}$ must satisfy the following boundary equation:

$\xi_{\Gamma}=\operatorname{Tr}_{\Gamma} P_{D_{1} \Gamma} \xi_{\Gamma}+\operatorname{Tr}_{\Gamma} \int_{D_{1}} G_{D}(\mathbf{z}, \mathbf{y}) f_{D_{1}}(\mathbf{y}) d \mathbf{y}$

The proof is based on the fundamental property of the potential: it is a projection $[15,16]$. It appears that the Eq. (9) does not depend on either $f_{D_{2}}$ or the boundary conditions outside $\overline{D_{1}}$.

From the general theory of the potentials [16] it follows that boundary equation (9) gives a necessary and sufficient condition for any boundary function $\xi_{\Gamma}$ to be smoothly extended from the boundary $\Gamma$ to the domain $D_{1}$. One should note here that, generally speaking, Eq. (9) is a vector equation since the clear trace is a vector-function.

In [14], the clear trace for the problem is obtained in a 1D case. Then, the Robin-type wall functions are derived via the theory of the Calderón-Ryaben'kii potentials.

Let us now extend the result of [14] to a multidimensional formulation. First, we introduce $\Gamma_{1}:=\bar{D}_{1} \cap \bar{D}_{2}$. Then, the clear trace is given by

$\operatorname{Tr}_{\Gamma} v_{D_{1}}=\left(l_{y} v(\mathbf{x}, 0), l_{\mathbf{x}} v_{\mid \gamma_{1}}, v\left(y^{*}\right), \frac{d v}{d y}\left(y^{*}\right)\right)^{T}$,

where $\gamma_{1}:=\partial \Omega \times[0 y *]$.

To prove this statement, we suppose that two arbitrary functions $v$ and $w$ from $\Xi_{D}$ have the same boundary vector (10). Then, let us consider function: $u_{D_{1}}=v_{D_{1}}-w_{D_{1}}$. The function $u_{D_{1}}$ satisfies homogeneous boundary conditions on $\Gamma$ and can smoothly be extended to the entire domain $D$ by the nil-function:

$u(\mathbf{z})= \begin{cases}u_{D_{1}} & \text { if } \mathbf{z} \in \bar{D}_{1}, \\ 0 & \text { if } \mathbf{z} \in D \backslash D_{1} .\end{cases}$

It is easy to see that $u \in \Xi_{D}$ and $\int_{D_{1}} G_{D}(\mathbf{z}, \mathbf{y}) L u d \mathbf{y}=\int_{D} G_{D}(\mathbf{z}, \mathbf{y}) L u d \mathbf{y}=$ $u(\mathbf{z})$. Thus, from the definition (6), it follows that

$P_{D_{1}} u_{D_{1}}=P_{D_{1}} v_{D_{1}}-P_{D_{1}} w_{D_{1}}=0_{D_{1}}$

Hence, the boundary vector (10) gives the clear trace.

The boundary equation (9) can be interpreted as the exact transfer of the boundary condition from the boundary $\Gamma \backslash \Gamma_{1}$ to the intermediate boundary $\Gamma_{1}$. It is easy to see that the intermediate boundary condition on the boundary $\Gamma_{1}$ is always nonlocal.
In the next Section, we obtain the nonlocal boundary condition transfer for a quite general case of BVP (3)-(5), which allows us to obtain the analytical solution.

\section{Analytical $D-t-N$ map}

Assume that in Eq. (3) $\gamma \leqslant 0$ and the boundary condition at $y=0$ :

$l_{y} U \equiv \omega_{1} U-\omega_{2} U_{y}$

$\omega_{1} \geqslant 0, \omega_{2} \geqslant 0$

$\omega_{1}+\omega_{2}>0$.

Let us consider a BVP for Eq. (3) in $[01] \times\left[0 y^{*}\right]$, which is supposed to be well-posed:

$\left(\mu(y) W_{y}\right)_{y}+\sigma(y) W_{x x}+\beta(y) W_{y}+\gamma(y) W=f(x, y)$

$l_{y} W(x, 0)=\alpha_{w}(x)$

$W(0, y)=U_{0}(y)$

$W(1, y)=U_{1}(y)$,

$W\left(x, y^{*}\right)=U^{*}(x)$.

Here and further, if we meet discontinuous boundary conditions, we seek a bounded solution.

It is clear that the equation in (12) can be rewritten as follows:

$\alpha W_{x x}-L_{y} W=\Phi$,

where

$-L_{y} W \stackrel{\text { def }}{=} W_{y y}+\widetilde{\beta}(y) W_{y}+\widetilde{\gamma} W=\frac{1}{p}\left(p W^{\prime}\right)^{\prime}+\widetilde{\gamma} W$,

where $\widetilde{\beta}=\left(\mu_{y}+\beta\right) \mu, \widetilde{\gamma}=\gamma / \mu, \Phi=f / \mu, p=\exp \left(\int_{0}^{y} \widetilde{\beta} d \xi\right)$.

One can prove that the operator $\widehat{L}_{y}:=p L_{y}$ is positive definite [18]. Hence, it is Hermitian.

Then, the general solution of BVP (12) can be obtained by the method of separation of variables [18]. One can show that the solution is given by the Fourier series:

$W(x, y)=\sum_{0}^{\infty} T_{k}(y) V_{k}(x)$

In (14), $T_{k}$ are determined by the Sturm-Liouville problem:

$\widehat{L}_{y} T_{k}=\rho \lambda_{k} T_{k}$,

$l_{y} T_{k}(0)=0$,

$T_{k}\left(y^{*}\right)=0$,

where $\rho=\alpha p$.

It is well-known that in the Sturm-Liouville problem $\lambda_{k} \geqslant 0$, and all $T_{k}$ are orthogonal to each other in the following scalar product: $(a, b)_{\rho}=\int_{0}^{y^{*}} \rho a b d y$.

Then, in (14), the Fourier coefficients are given by

$V_{k}(x)=\int_{0}^{y^{*}} \rho W(x, y) T_{k}(y) d y=\left(W, T_{k}\right)_{\rho}$,

where $\left(T_{k}, T_{k}\right)_{\rho}=1$.

Multiplying (13) by $\rho T_{k}$ in the sense of the scalar product, one can obtain a BVP for $V_{k}$ :

$V_{k}^{\prime \prime}-\lambda_{k} V_{k}=c_{k}$,

$V_{k}(0)=a_{k}, V_{k}(1)=b_{k}$,

where $c_{k}=\frac{1}{\alpha}\left(\Phi, T_{k}\right)_{\rho}, a_{k}=\left(U_{0}, T_{k}\right)_{\rho}, b_{k}=\left(U_{1}, T_{k}\right)_{\rho}$.

The Green's function of BVP (16) is given by:

$\bar{G}_{k}\left(x, \xi, \lambda_{k}\right)=\frac{1}{J(1)} \times \begin{cases}\operatorname{sh}\left(\sqrt{\lambda_{k}} x\right) \operatorname{sh}\left(\sqrt{\lambda_{k}}(1-\xi)\right), & 0 \leqslant x \leqslant \xi, \\ \operatorname{sh}\left(\sqrt{\lambda_{k}}(1-x)\right) \operatorname{sh}\left(\sqrt{\lambda_{k}} \xi\right), & \xi \leqslant x \leqslant 1,\end{cases}$

where $J(x)=J(1)$ is the Wronskian and $J(1)=-\sqrt{\lambda_{k}} \operatorname{sh}\left(\sqrt{\lambda_{k}}\right)$. 
Taking into account that the Green's function of the operator $\widehat{L}_{y}$ in (15) is the following:

$\widetilde{G}_{n}(y, \eta)=\frac{1}{\lambda_{n}} T_{n}(y) T_{n}(\eta)$. (12):

One can obtain the general Green's function of the original BVP

$G(x, \xi, y, \eta)=p(\eta) \sum_{0}^{\infty} T_{k}(y) T_{k}(\eta) \bar{G}_{k}\left(x, \xi ; \lambda_{k}\right)$.

Then, the general solution of BVP (12) at any internal point is given by:

$$
\begin{aligned}
W= & \int_{0}^{1} \int_{0}^{y^{*}} G(x, y, \xi, \eta) \Phi(\xi, \eta) d \xi d \eta-\alpha \int_{0}^{y^{*}} U_{0} G_{\xi \mid \xi=0} d \eta \\
& +\alpha \int_{0}^{y^{*}} U_{1} G_{\xi \mid \xi=1} d \eta-\int_{0}^{1} \alpha_{w} G_{\mid \eta=0} d \xi+\int_{0}^{1} U^{*} G_{\eta \mid \eta=y^{*}} d \xi .
\end{aligned}
$$

One can show that the solution (18) is represented by a doublelayer potential with the density $U^{*}$. Its derivative $W_{y}$ is continuous up to the boundary $y=y^{*}$.

Hence,

$$
\begin{aligned}
W_{y \mid y=y^{*}}= & \int_{0}^{1} \int_{0}^{y^{*}} G_{y \mid y=y^{*}} \Phi(\xi, \eta) d \xi d \eta-\alpha \int_{0}^{y^{*}} U_{0} G_{y \xi \mid \xi=0, y=y^{*}} d \eta \\
& +\alpha \int_{0}^{y^{*}} U_{1} G_{y \xi \mid \xi=1, y=y^{*}} d \eta-\int_{0}^{1} \alpha_{w} G_{y \mid \eta=0, y=y^{*}} d \xi \\
& +\int_{0}^{1} U^{*} G_{y \eta \mid \eta=y^{*}, y=y^{*}} d \xi .
\end{aligned}
$$

The operator on the right-hand side of (19) can be interpreted as an operator providing a $D-t-N$ map: $U^{*} \mapsto U_{y}^{*}=W_{y \mid y=y^{*}}$. Thus,

$$
\begin{aligned}
U_{y}^{*}= & \int_{0}^{1} \int_{0}^{y^{*}} G_{y \mid y=y^{*}} \Phi(\xi, \eta) d \xi d \eta-\alpha \int_{0}^{y^{*}} U_{0} G_{y \xi \mid \xi=0, y=y^{*}} d \eta \\
& +\alpha \int_{0}^{y^{*}} U_{1} G_{y^{\xi} \mid \xi=1, y=y^{*}} d \eta-\int_{0}^{1} \alpha_{w} G_{y \mid \eta=0, y=y^{*}} d \xi \\
& +\int_{0}^{1} U^{*} G_{y \eta \mid \eta=y^{*}, y=y^{*}} d \xi .
\end{aligned}
$$

Let us set

$U^{*}=U\left(x, y^{*}\right)$,

where $U$ is the solution of BVP (3)-(5).

Then, from the uniqueness of the solution of BVP (12), we have $U_{y}^{*}=U_{y}\left(x, y^{*}\right)$. Eq. (20) corresponds to the second equation in (9). One should note that the first equation in (9) is automatically satisfied due to the definition of the $D-t-N$ map.

Thus, Eq. (19) gives us the boundary condition on the intermediate boundary $y=y^{*}$. It is easy to see that the boundary condition (19) is nonlocal. One should note that Eq. (19) does not depend, for example, on the boundary condition (5). If the function $U_{s}$ changes, then both $U^{*}$ and $U_{y}^{*}$ can change but their relation given by the Eq. (19) retains.

\section{Numerical $D-t-N$ map}

In a general case, we are obviously not able to obtain the Green's function and represent the $D-t-N$ map via the integral equation. However, the required $D-t-N$ map can be obtained numerically.

Next, in the domain $D_{1}$ we consider the BVP similar to (12):

$L W=f$,

$l_{y} W(\mathbf{x}, 0)=\alpha_{w}(\mathbf{x})$,

$l_{\mathbf{x}} W_{\mid \gamma_{1}}=\beta(y)$,

$W\left(\mathbf{x}, y^{*}\right)=U^{*}$.
Then, we consider the following $D-t-N$ map:

$U_{\mathbf{n}}^{a s t}=\mathfrak{R}_{\Gamma_{1}} U^{*}$.

Here, $\mathfrak{R}_{\Gamma_{1}}: U^{*} \rightarrow U_{\mathbf{n}}^{*}=W_{y \mid y=y^{*}}$ is the Poincaré-Steklov operator determined by BVP (22), in which $U^{*}(\mathbf{x})$ is an input function. Thus, the pseudo-differential operator $\mathfrak{R}_{\Gamma_{1}}$ is determined by the derivative $W_{y}\left(\mathbf{x}, y^{*}\right)$ :

$\mathfrak{R}_{\Gamma_{1}} U^{*} \equiv W_{y}\left(\mathbf{x}, y^{*} ; U^{*}\right)$.

It is easy to see that the operator $\mathfrak{R}_{\Gamma_{1}}$ is linear and acts nonlocally on the entire boundary $\Gamma_{1}$ because its application requires the solution of BVP (25) in the domain $D_{1}$, which depends on the entire function $U^{*}(\mathbf{x})$.

Similar to the previous consideration, the intermediate boundary condition at $\Gamma_{1}: y=y^{*}<1$ can be given by the boundary equation (23). Yet the calculation of the operator $\mathfrak{R}_{\Gamma_{1}}$ requires the solution of BVP (22). Our task is to obtain a representation of $\mathfrak{R}_{\Gamma_{1}} U^{*}$ fast enough to make the domain decomposition efficient.

For further consideration, we represent the solution of BVP (1) and (2) on the domain $D_{1}$ as a sum: $W=W_{e}+W_{i}$, where $W_{e}$ and $W_{i}$ are the solutions of the following BVPs:

$L W_{e}=f$,

$l_{y} W_{e}(\mathbf{x}, 0)=\alpha_{w}(\mathbf{x})$

$l_{\mathbf{x}} W_{e \mid \gamma_{1}}=\beta(y)$,

$W_{e}\left(\mathbf{x}, y^{*}\right)=0$,

and

$L W_{i}=0$,

$l_{y} W_{i}(\mathbf{x}, 0)=0$,

$l_{\mathbf{x}} W_{i \mid \gamma_{1}}=0$,

$W_{i}\left(\mathbf{x}, y^{*}\right)=U^{*}$.

It is clear that the function $W_{e}$ yields the explicit part of the solution of BVP (22) that does not depend on $U^{*}$.

\subsection{Separation of variables}

First, let us consider the case of separation of variables. Assume that $L:=\widehat{L}_{\mathbf{x}}+\widehat{L}_{y}$, where $\widehat{L}_{\mathbf{x}}$ and $\widehat{L}_{y}$ are operators depending on $\mathbf{x}$ and $y$, respectively. Suppose that $\widehat{L}_{\mathbf{x}}$ is positive definite. Then, the operator $\mathfrak{R}_{\Gamma_{1}}$ can explicitly be obtained by the Fourier method of separation of variables. Indeed, in this case, the eigenvalues $\lambda_{k}(k=0,1, \cdots)$ of the operator $\widehat{L}_{\mathbf{x}}$ are positive and its eigenfunctions $\phi_{k}(k=0,1, \cdots)$ are orthogonal to each other. In addition, the solution of BVP (22) can be represented by a regularly converging Fourier series.

We can always choose the eigenfunctions and their numeration in such a way that

$0 \leqslant \lambda_{0} \leqslant \lambda_{1} \leqslant \cdots$

and $\left(\phi_{n}, \phi_{n}\right)=1,(n=0,1, \cdots)$, where $(\cdot, \cdot)$ is some scalar product.

We seek the general solution of (25) in the form of the Fourier series:

$W_{i}(\mathbf{x}, y)=\sum_{0}^{\infty} \widehat{u}_{n}^{*} v_{n}(y) \phi_{n}(\mathbf{x})$,

where $\widehat{u}_{n}^{*}=\left(U^{*}, \phi_{n}\right), v_{n}\left(y^{*}\right)=1,(n=0,1, \cdots)$.

Substituting (26) into (25), we obtain a BVP for each $v_{n}$ :

$\widehat{L}_{n} v_{n}=0$,

$l_{y} v_{n}(0)=0$,

$v_{n}\left(y^{*}\right)=1$.

Here, $\widehat{L}_{n}:=\widehat{L}_{y}+\lambda_{n} I, I$ is the unit operator. 
Then, since the system of functions $\phi_{n}(n=0,1,2, \ldots)$ is full,

$$
\begin{aligned}
W_{i \mid y}^{*}\left(\mathbf{x}, y^{*}\right) & =\sum_{0}^{\infty} \widehat{u}_{n}^{*} v_{0}^{*}\left(y^{*}\right) \phi_{n}(\mathbf{x})+\sum_{1}^{\infty} \widehat{u}_{n}^{*}\left(v_{n}^{\prime}\left(y^{*}\right)-v_{0}^{\prime}\left(y^{*}\right)\right) \phi_{n}(\mathbf{x}) \\
& =U^{*}(\mathbf{x}) v_{0}^{\prime}\left(y^{\prime}\right)+\sum_{1}^{\infty} \widehat{u}_{n}^{*}\left(v_{n}^{\prime}\left(y^{*}\right)-v_{0}^{\prime}\left(y^{*}\right)\right) \phi_{n}(\mathbf{x}) .
\end{aligned}
$$

Thus, we obtain that

$$
\mathfrak{R}_{\Gamma_{1}} U^{*}=U^{*}(\mathbf{x}) v_{0}^{\prime}\left(y^{*}\right)+\sum_{1}^{\infty} \widehat{u}_{n}^{*}\left(v_{n}^{\prime}\left(y^{*}\right)-v_{0}^{\prime}\left(y^{*}\right)\right) \phi_{n}(\mathbf{x})+W_{e \mid y}^{\prime}\left(\mathbf{x}, y^{*}\right) .
$$

\subsection{General case}

In the general case, let us introduce a set of basis functions $s_{n}(\mathbf{x})$. Then, we consider the following set of appropriate BVPs:

$$
\begin{aligned}
& L w_{n}=0, \\
& l_{y} w_{n}(\mathbf{x}, 0)=0, \\
& l_{\mathbf{x}} w_{n \mid \gamma_{1}}=0, \\
& w_{n}\left(\mathbf{x}, y^{*}\right)=s_{n}(\mathbf{x}) .
\end{aligned}
$$

Next, we construct the operator $\mathfrak{R}_{\Gamma_{1}}$ as follows. In the domain $D_{1}$, the input function $U^{*}$ is approximated as

$U^{*}(\mathbf{x}) \approx \sum_{n=0}^{N} u_{n}^{*} s_{n}(\mathbf{x})$,

where $N \geqslant 0$, the coefficients $u_{n}^{*}$ are determined by variational problem:

$\min _{u_{n}^{*}}\left\|U^{*}(\mathbf{x})-\sum_{0}^{N} u_{n}^{*} s_{n}(\mathbf{x})\right\|_{L_{2}}$.

Then, the function $W_{i}$ is approximated by the set of functions $w_{n}$ :

$W_{i}(\mathbf{x}, y) \approx \sum_{0}^{N} u_{n}^{*} w_{n}(\mathbf{x}, y)$

If $s_{0} \equiv 1$ and $N \geqslant 1$, then approximation (32) can be rewritten as:

$$
\begin{aligned}
W_{i}(\mathbf{x}, y) & \approx \sum_{0}^{N} u_{n}^{*} w_{n}(\mathbf{x}, y)+\left(U^{*}(\mathbf{x})-\sum_{0}^{N} u_{n}^{*} s_{n}(\mathbf{x})\right) w_{0}(\mathbf{x}, y) \\
& =U^{*}(\mathbf{x}) w_{0}(\mathbf{x}, y)+\sum_{1}^{N} u_{n}^{*}\left(w_{n}(\mathbf{x}, y)-s_{n}(\mathbf{x}) w_{0}(\mathbf{x}, y)\right) .
\end{aligned}
$$

One should note that if $N=0$, then we arrive at the following simple approximation:

$W_{i}(\mathbf{x}, y) \approx U^{*}(\mathbf{x}) w_{0}(\mathbf{x}, y)$.

Thus, the derivative $W_{i \mid y}^{\prime}\left(\mathbf{x}, y^{*}\right)$ is represented similar to the expansion (28):

$W_{i \mid y}^{\prime}\left(\mathbf{x}, y^{*}\right) \approx U^{*}(\mathbf{x}) w_{0}^{\prime}\left(\mathbf{x}, y^{*}\right)+\sum_{1}^{N \geqslant 1} u_{n}^{*}\left(w_{n}^{\prime}\left(\mathbf{x}, y^{*}\right)-s_{n}(\mathbf{x}) w_{0}^{\prime}\left(\mathbf{x}, y^{*}\right)\right)$.

Hence,

$$
\begin{aligned}
\Re_{\Gamma_{1}} U^{*} \approx & U^{*}(\mathbf{x}) w_{0}^{\prime}\left(y^{*}\right)+\sum_{1}^{N \geqslant 1} u_{n}^{*}\left(w_{n}^{\prime}\left(\mathbf{x}, y^{*}\right)-s_{n}(\mathbf{x}) w_{0}^{\prime}\left(\mathbf{x}, y^{*}\right)\right) \\
& +W_{e \mid y}^{\prime}\left(\mathbf{x}, y^{*}\right) .
\end{aligned}
$$

\section{Domain decomposition}

Let us set $U^{*}(\mathbf{x})=U\left(\mathbf{x}, y^{*}\right)$ in (22), where $U$ is the solution of BVP (1) and (2) to be found. Then, Eq. (23) yields:

$U_{y \mid y=y^{*}}^{\prime}=\mathfrak{R}_{\Gamma_{1}} U\left(\mathbf{x}, y^{*}\right)$.

The solution of BVP (22) is unique. Hence, $W=U_{D_{1}}$.

The pseudo-differential equation (36) can be interpreted as the exact boundary condition on the intermediate boundary $y=y^{*}$ for the BVP in the domain $D_{2}$. Yet the use of this boundary condition is not straightforward because the function $U\left(\mathbf{x}, y^{*}\right)$ is unknown. It is clear that $U\left(\mathbf{x}, y^{*}\right)$ cannot be determined in the framework of a BVP formulated only on $D_{1}$. We obtain this function as soon as the BVP on $D_{2}$ is solved with the appropriate intermediate boundary condition (36).

Thereby, we arrive at the following domain decomposition. In the external domain $D_{2}$ we have a BVP:

$L V=f$,

$l_{\mathbf{x}} V_{\mid \gamma_{2}}=\beta(y)$

$V(\mathbf{x}, 1)=U_{s}(\mathbf{x})$

$V_{y}\left(\mathbf{x}, y^{*}\right)=\mathfrak{R}_{\Gamma_{1}} V$,

where $\gamma_{\mid 2}:=\partial \Omega \times\left[y^{*} 1\right]$.

Hence, the BVP (37) and (38) is fully determined. Then, we are able to solve BVP (22) with $U^{*}=V\left(\mathbf{x}, y^{*}\right)$. From the uniqueness of the solution it follows that $V=U_{D_{2}}$ and $W=U_{D_{1}}$.

The solution of BVP (37) and (38) involves a function $\mathfrak{R}_{\Gamma_{1}} V\left(\mathbf{x}, y^{*}\right)$. It cannot be calculated immediately since $V\left(\mathbf{x}, y^{*}\right)$ is unknown. Under some conditions an iterative process can be organized. On this way, the nonlocal term of (38) is taken from the previous iteration. Meanwhile, such kind of a domain decomposition is not usually efficient. In a 1D case, it is equivalent to the realization of a Robin boundary condition via an iterative procedure. Even if the both sides of the Eq. (38) are considered simultaneously, the decomposition may not be efficient unless a special consideration of the operator $\mathfrak{R}_{\Gamma_{1}}$ is used.

One can note that by the use of the spectral approach described above, the pseudo-differential operator in (38) is explicitly represented by (35). It is important to emphasize here that, in (35), the functions $w_{n}$ and $W_{e}$ do not depend on the BVP in the outer domain and can be found in advance from the solution of BVPs (30) and (24), respectively. Thus, these functions are to be calculated only once. Then, outside the domain $D_{1}$, they can be used for very different boundary conditions, the right-hand side $f$ and domain $D_{2}$. A similar statement is applicable to the Eqs. (29) and (27).

Meanwhile, the solution of BVP (37) still retains complicated because the expansion coefficients $u_{n}^{*}$ are unknown. They should be found from Eq. (31) as the BVP is solved. In the case of explicit numerical methods, the Eq. (31) can be used straightforward to provide the solution at the boundary nodes via the solution at the internal nodes nearest to the boundary. If an implicit algorithm is used, the appropriate matrix becomes ill-structured.

A possible way to overcome the problem is the following. One can note that

$\mathfrak{R}_{\Gamma_{1}} U^{*}=\mathfrak{R}_{\Gamma_{1}} U^{*}\left(\mathbf{x}^{*}\right)+\mathfrak{R}_{\Gamma_{1}}\left(U^{*}-U^{*}\left(\mathbf{X}^{*}\right)\right), \quad \mathbf{x}^{*} \in \Gamma_{1}$.

Then, the boundary condition (38) at any point $\mathbf{x}^{*} \in \Gamma_{1}$ can be rewritten as:

$V_{y}^{\prime}\left(\mathbf{x}^{*}, y^{*}\right)=V\left(\mathbf{x}^{*}, y^{*}\right) \Re_{\Gamma_{1}}(1)_{\mid x=x^{*}}+\Re_{\Gamma_{1}}\left(V-V\left(\mathbf{x}^{*}, y^{*}\right)\right)_{\mid x=x^{*}}$.

Although the first term in (39) is nonlocal, it does not depend on the expansion coefficients. It corresponds to both the first and last terms in (35). One can consider the first term in (39) as the "diagonal" and take it from a current iteration, while the second term is 
taken from the previous iteration. Some justification to this assumption will be given in the next Section. In this case, the expansion coefficients $u_{n}^{*}$ in (31) are calculated on the basis of the solution at the previous iteration. Thus, at each iteration, we have a Robin boundary condition. The iterations can be included in an iterative procedure required for solving the elliptic BVP on $D_{2}$. One should note that in turbulent flows the external problem is always nonlinear, and the appropriate iterations, related to the nonlinearity, are necessary. Yet a high-accuracy decomposition is not probably justified in this case because of the required linearization of the governing equations in the near-wall area (domain $D_{1}$ ) and an inevitable error caused by that. It is important to note that in the algorithm developed we have not required the linearity of the problem on the domain $D_{2}$.

In the most general case, we can carry out the transfer of the boundary condition via the Difference Potential Method by Ryaben'kii (DPM) [15]. In the DPM a BVP is first reduced to a boundary equation. Therefore, the expansion coefficients can be found in a natural way as the boundary equation is solved. It is to be noted here that the DPM can efficiently be applied for the problems with complex geometries.

\section{Nonlocal wall function}

Let us retain only the first term on the right-hand side in (39). Then, we arrive at the following intermediate boundary condition: $V_{y}^{\prime}\left(\mathbf{x}, y^{*}\right)=V\left(\mathbf{x}, y^{*}\right) w_{0 \mid y}^{\prime}\left(\mathbf{x}, y^{*}\right)+W_{e \mid y}^{\prime}\left(\mathbf{x}, y^{*}\right)$.

Here, $W_{e}$ is obtained from (24), while $w_{0}$ is determined by BVP:

$$
\begin{aligned}
& L w_{0}=0, \\
& l_{y} w_{0}(\mathbf{x}, 0)=0, \\
& l_{\mathbf{x}} w_{0 \mid \gamma_{1}}=0, \\
& w_{0}\left(\mathbf{x}, y^{*}\right)=1 .
\end{aligned}
$$

Thus, we arrive at the intermediate boundary condition of Robintype, which does not contain any expansion coefficients $u_{n}$. However, it captures some nonlocal effects because they include the functions $W_{e}(\mathbf{x}, y)$ and $w_{0}(\mathbf{x}, y)$. Therefore, we shall interpret such a boundary condition as a nonlocal wall function. More precisely, under the wall function we understand any function from space $\Xi_{\Gamma}$, which satisfies the boundary equation (40). It is important to underline that both the functions $W_{e}$ and $w_{0}$ do not depend on the solution on the external domain $D_{2}$, and they can be calculated in advance.

One can note that for Eq. (24) the boundary conditions in $\mathbf{x}$ direction can be unknown. Then, if we assume that the boundaries are situated in the areas of weak enough dependence of the solution on $x$, it is natural to set the homogeneous Newmann boundary conditions. In addition, the error generated by these approximate boundary conditions should be small if the near-wall domain $D_{1}$ is thin enough.

Thus, the solution $V$ in the domain $D_{2}$ is obtained with the boundary condition (40). Then, in the domain $D_{1}$ we immediately have:

$W(\mathbf{x}, y)=V\left(\mathbf{x}, y^{*}\right) w_{0}(\mathbf{x}, y)+W_{e}(\mathbf{x}, y)$.

In the turbulent flow modeling, we often require fluxes to a wall. They can be obtained from Eq. (41) at once because both the functions $w_{0}$ and $W_{e}$ are known in advance:

$W_{y}^{\prime}(\mathbf{x}, 0)=V\left(\mathbf{x}, y^{*}\right) w_{0 \mid y}^{\prime}(\mathbf{x}, 0)+W_{e \mid y}^{\prime}(\mathbf{x}, 0)$.

One should note that if we simplify the operator $L$ setting $\alpha=0$ in (3): $L:=\mu L_{y}$, then the operator $\mathfrak{R}_{\Gamma_{1}}$ becomes local. In this case, the operator $\mathfrak{R}_{\Gamma_{1}}$ is only based on the solution of a BVP with respect to the variable $y$. Then, the nonlocal function (40) is reduced to the Robin-type wall function [14]:
$V_{y}^{\prime}\left(\mathbf{x}, y^{*}\right)=V\left(\mathbf{x}, y^{*}\right) \widetilde{w}_{0}^{\prime}\left(y^{*}\right)+\widetilde{W}_{e \mid y}^{\prime}\left(\mathbf{x}, y^{*}\right)$.

Here, $\widetilde{w}_{0}$ is obtained from a $1 \mathrm{D}$ BVP:

$L_{y} \widetilde{w}_{0}=0$,

$l_{y} \widetilde{w}_{0}(0)=0$,

$\widetilde{w}_{0}\left(y^{*}\right)=1$,

while the function $\widetilde{W}_{e}$ is determined by a 1D BVP:

$\mu L_{y} \widetilde{W}_{e}=f$,

$l_{y} \widetilde{W}_{e}(\mathbf{x}, 0)=0$,

$\widetilde{W}_{e}\left(\mathbf{x}, y^{*}\right)=0$.

Obviously, in this case the solution in the domain $D_{1}$ is given by:

$W(\mathbf{x}, y)=V\left(\mathbf{x}, y^{*}\right) \widetilde{w}_{0}(\mathbf{x}, y)+\widetilde{W}_{e}(\mathbf{x}, y)$.

In the next Section, we consider an example of BVP (3)-(5). The numerical solution is obtained both in the entire single domain and by the domain decomposition. The domain decomposition is realized via the exact and approximate decomposition approaches. In the latter case, the 1D and nonlocal wall functions are tested on very different solutions. As the 1D wall function [10], we use the boundary condition (42). In this case, the boundary condition is exactly transferred to the intermediate boundary provided that the derivatives with respect to $x$ are neglected.

\section{Numerical nonlocal wall functions and decomposition method}

Assume that in BVP (3)-(5) $\epsilon=3 \cdot 10^{-2}, \delta=10^{-2}, \beta=y, \gamma=$ $-y^{2}, f=10^{2} x^{2}, U_{s}=1+10^{2} x^{2}, \alpha_{w}=0, l_{y}:=1$.

The profile of the "viscosity" coefficient $\mu$ corresponding to these data is shown in Fig. 1. The coefficient rapidly changes from a relatively small "laminar" viscosity $\mu_{l}=\delta /$ Re to a much bigger "turbulent" viscosity $\mu_{t} \approx 1 / R e$.

In the boundary conditions (4), the functions $U_{0}$ and $U_{1}$ are chosen in such a way that they satisfy the following 1D BVP:

$\left(\mu(y) v_{y}\right)_{y}+\beta v_{y}+\gamma v=0$,

$v(0)=0, v(1)=U_{s}$.

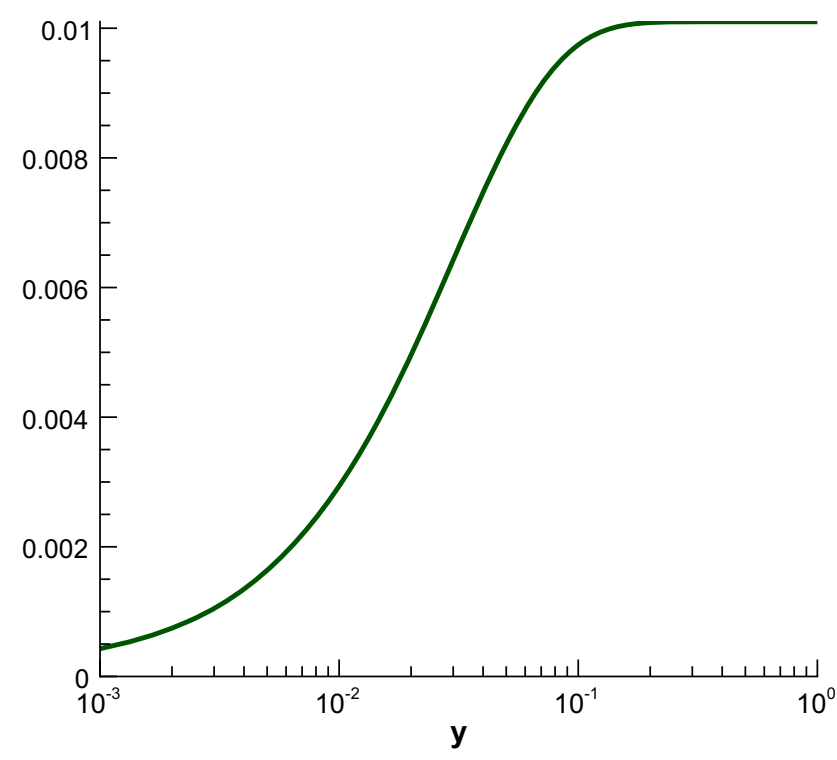

Fig. 1. Profile of $\mu$. 
Such boundary conditions mean that the flow becomes locally $1 \mathrm{D}$ in a vicinity of the boundaries $x=0$ and $x=1$. Condition (43) models a near-wall flow in which nonlocal effects due to the source term and wall curvature are only localized in some domain.

It is clear that in BVP (3)-(5), (43) the nonlocal effects are determined by the value of the parameter $\alpha$. Further, the domain decomposition method as well as the 1D and nonlocal wall functions are tested for two very different values of $\alpha$. The computational domain is split into two subdomains at point $y^{*}=$ $0.1:\left[\begin{array}{ll}0 & 1\end{array}\right]:=\left[\begin{array}{ll}0 & 0.1\end{array}\right] \cup[0.11]$.

If $\alpha=1$, then the term with $U_{x x}$ asymptotically vanishes if $R e$ tends to infinity. Here, there is an immediate analogue with a classical boundary-layer type flow. In this case, the 1D wall-function approach (42) gives a rather good prediction of the solution. In Fig. 2, the profile of the solution at point $x=0.8$ is shown. In this plot and further, the solid line corresponds to the "exact" solution

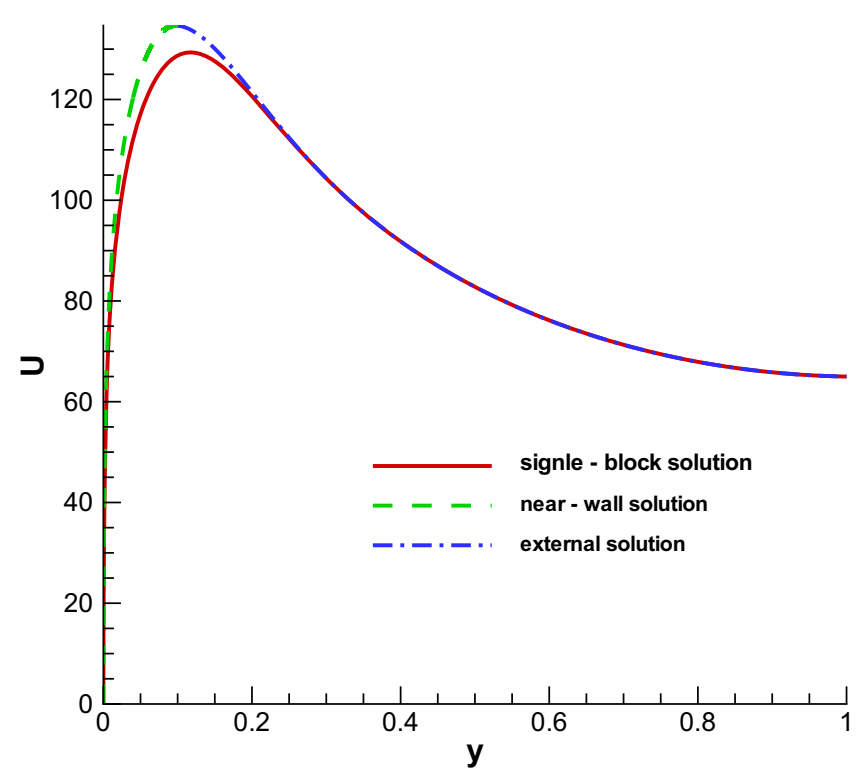

Fig. 2. 1D wall function. Profile of $U$ at $x=0.8, \alpha=1$.

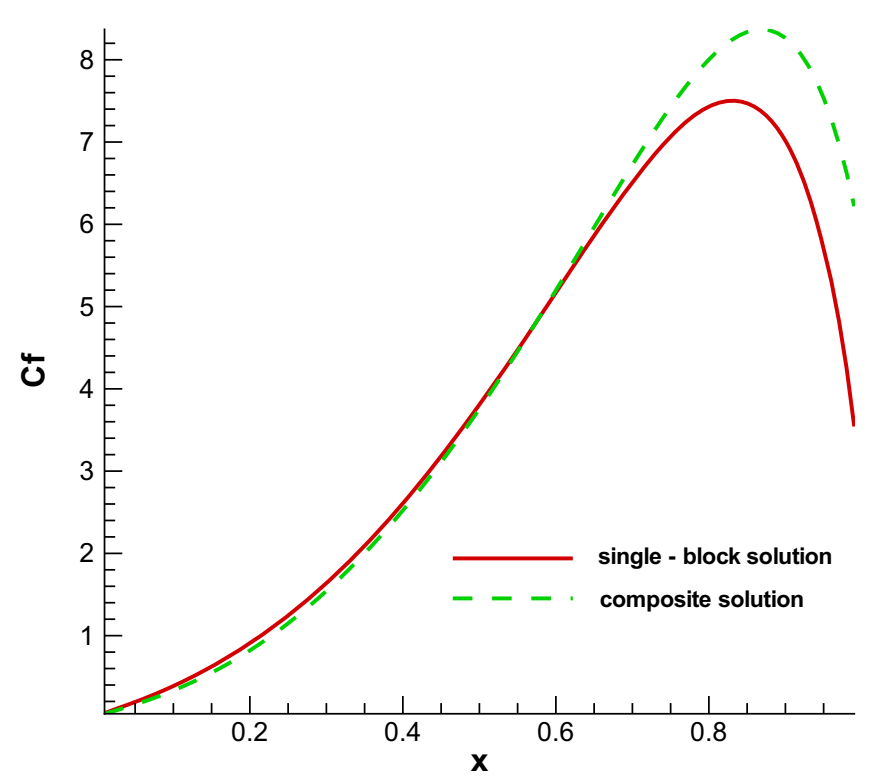

Fig. 3. 1D wall function. Distribution of $C_{f}, \alpha=1$. obtained on a single domain using a fine enough mesh. In the composite profile, the dashed line represents the near-wall solution, while the dash-dotted line shows the solution obtained in the outer domain. The prediction of the friction coefficient $C_{f}=\mu \frac{\partial U}{\partial y}(x, 0)$ is also reasonably good and given in Fig. 3 .

If $\alpha$ significantly increases, then the 1D wall function (42) becomes unacceptable. For $\alpha=10$, it is demonstrated by the solution profile $U$ at the point $x=0.8$ and the distribution of the coefficient $C_{f}$ in Figs. 4 and 5, respectively.

The nonlocal-wall-function approach (41) gives a much better prediction. In this case, the profile of $U$ almost coincides with the exact solution. Meanwhile, the approximation of the friction coefficient $C_{f}$ is also quite good. It is shown in Fig. 6.

The use of trigonometrical functions as the basis functions in (33):

$s_{2 n-1}=\sin (2 \pi n x), s_{2 n}=\cos (2 \pi n x)(n=0, \ldots, N)$,

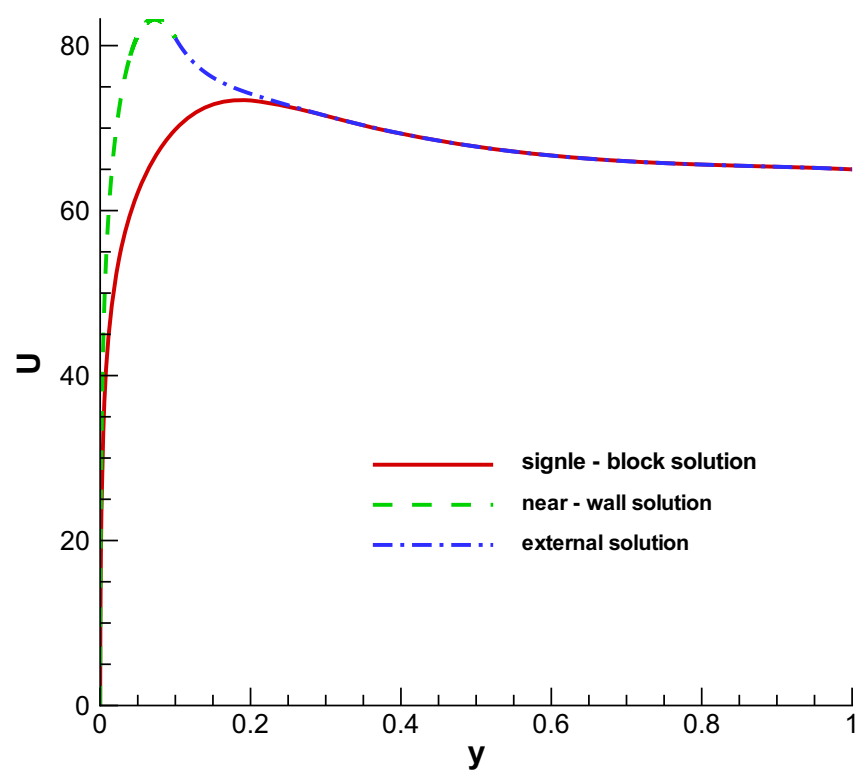

Fig. 4. 1D wall function. Profile of $U$ at $x=0.8, \alpha=10$.

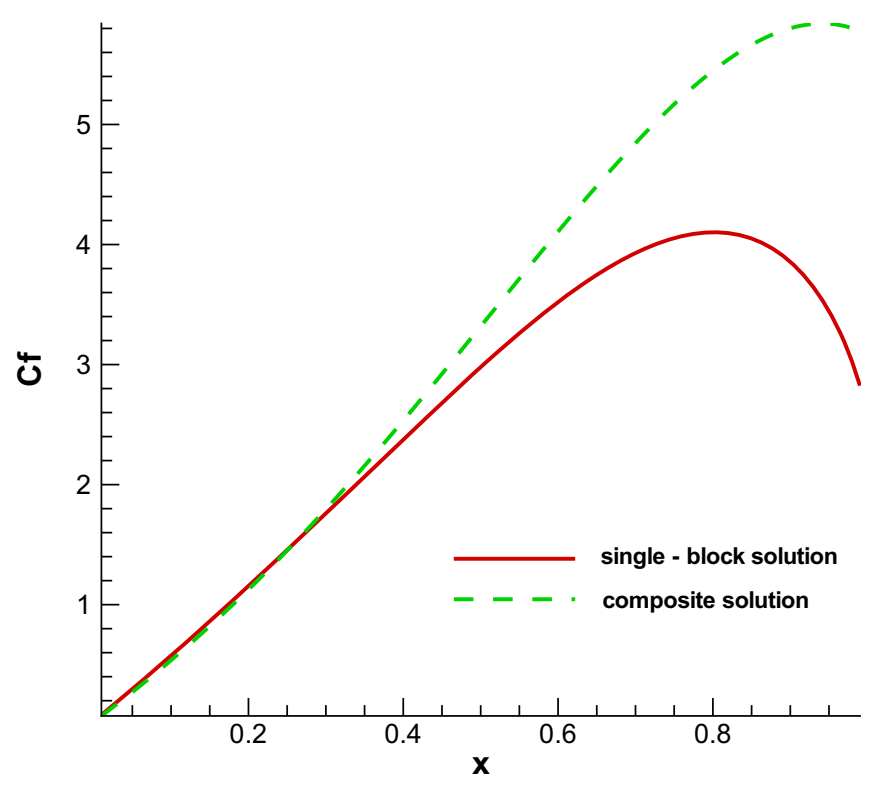

Fig. 5. $1 \mathrm{D}$ wall function. Distribution of $C_{f}, \alpha=10$. 


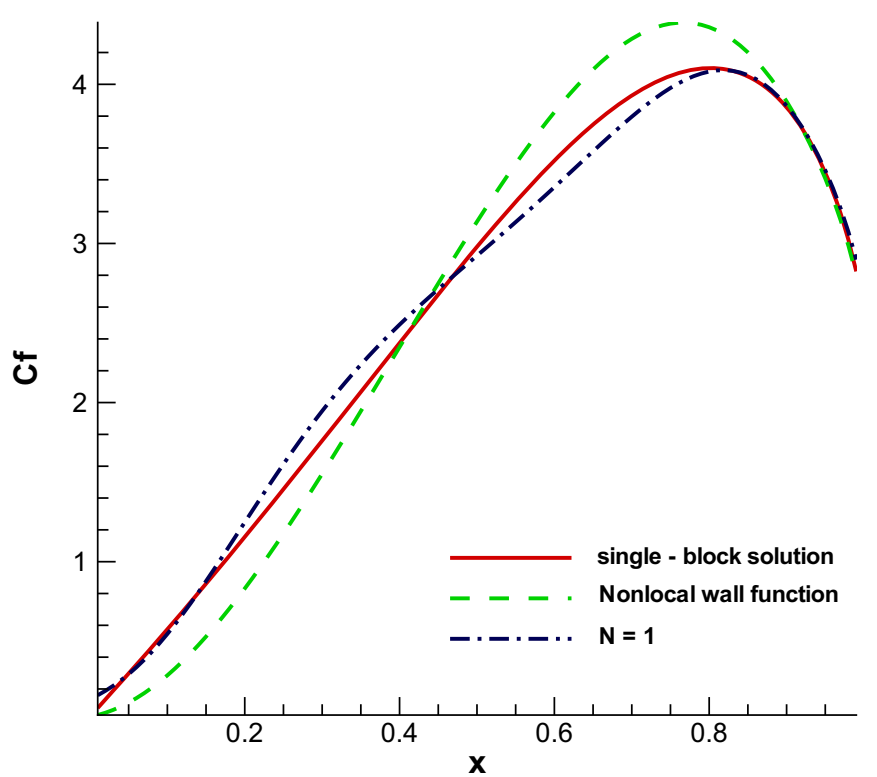

Fig. 6. Nonlocal wall function and domain decomposition. Distribution of $C_{f}, \alpha=10$.

results in the domain decomposition method. Taking into account the first harmonics $(N=1)$ already provides a good enough prediction as shown by the dash-dotted line in Fig. 6 . In the case of $N=2$, the profile of $C_{f}$ almost coincides with the exact solution.

In the example considered, it is important to note that the boundary condition (41) at the intermediate boundary does not depend on the solution in the external domain. Thus, the same boundary condition (nonlocal wall-function) can be used for very different external boundary conditions, e.g., the function $U_{s}$ in (5). It is clear that the computational time required for the solution of the BVP in the interval $\left[y^{*} 1\right]$ is significantly less than in the original domain because it does not include areas with high gradients of the solution. The efficiency of the approach assumes, of course, some serial computations are to be required for the problem with different external conditions. However, it usually occurs in the modeling of turbulent flows. In general, the same conclusion retains if we use more the complicated intermediate boundary condition (38) and (35). In this case, the calculation of several functions $w_{n}^{\prime}\left(x, y^{*}\right)$ is required.

\section{Conclusion}

For the analysis of domain-decomposition approaches to nearwall turbulent flows, a model 2D equation has been suggested. The equation has a high-gradient near-boundary profile and simulates RANS equations at high-Reynolds-numbers. A domain decomposition method has been developed. It allows one to mostly resolve the near-wall layer independently on the outer domain. Thereby, in the suggested approach the boundary conditions are transferred in advance from the wall to some intermediate boundary. The boundary conditions on the intermediate boundary can be realized in a separate routine. The method allows one to avoid a time-consuming near-wall resolution. A concept of nonlocal wall functions has been suggested. The nonlocal wall functions take into account flow properties along the wall and can be important in the case of complex geometries and significant local source terms.

One should note that the generalization of the developed technique to 3D problems is straightforward. The implementation of the method to RANS equations requires the development of some linear model near a wall (domain $D_{1}$ ). It can be done via the approximation of the efficient viscosity coefficient and convective terms similar to the analytical and Robin-type wall functions $[5,6,14]$. In contrast to these approaches, the developed technique is nonlocal and requires the solution of either 2D or 3D auxiliary BVPs.

In the future work, the approach will be implemented in RANS and LES.

\section{References}

[1] Kopp T. On grid-independence of RANS predictions for aerodynamic flows using model-consistent universal wall-functions. In: Proceedings of ECCOMAS CFD'2006. The Netherlands: 2006.

[2] Travin A, Shur M, Strelets M, Spalart PR. Detached-Eddy simulations past a circular cylinder. Flow Turbul Combust 2000;63:293-313.

[3] Launder BE, Spalding BI. The numerical computation of turbulent flows. Comput Methods Appl Mech Eng 1974;3:269-89.

[4] Craft TJ, Gant SE, Gerasimov AV, Iacovides H, Launder BE. Wall-function strategies for use in turbulent flow CFD. In: Proceedings of 12th International Heat Transfer Conference. Grenoble, France: 2002. p. 1-12.

[5] Craft TJ, Gerasimov AV, Iacovides H, Launder BE. Progress in the generalization of wall-functions treatments. Heat Fluid Flow 2002;23(2):148-60.

[6] Craft TJ, Gant SE, Iacovides H, Launder BE. A new wall function strategy for complex turbulent flows. Numer Heat Transfer 2004;45:301-18.

[7] Esch T, Menter FR. Heat transfer predictions based on two-equation turbulence models with advanced wall treatment. In: Proceedings of the 4th International Symposium on Turbulence, Heat \& Mass Transfer. Antalya, Turkey: 2003. p. 614-21.

[8] Wilcox DC. Wall matching, a rational alternative to wall functions AIAA Paper 89-611. Reno, NV: 1989.

[9] Kalitzin G, Medic G, Iaccarino G, Durbin PA. Near-wall behavior of RANS turbulence models and implications for wall function. J Comput Phys 2005;1:265-91.

[10] Utyuzhnikov SV. Some new approaches to building and implementation of wall-functions for modeling of near-wall turbulent flows. Comput Fluids 2005;34(7):771-84.

[11] Rubin SG, Tannehill JC. Parabolized/reduced Navier-Stokes computational techniques. Ann Rev Fluid Mech 1992;24:117-44.

[12] Utyuzhnikov SV. Generalized wall-functions and their application for simulation of turbulent flows. Int J Numer Methods Fluid 2005;47(1011):1323-8.

[13] Utyuzhnikov SV. The method of boundary condition transfer in application to modeling near-wall turbulent flows. Comput Fluids 2006;35(10):1193-204.

[14] Utyuzhnikov SV. Robin-type wall functions and their numerical implementation. J Appl Numer Math 2008;58(10):1521-33.

[15] Ryaben'kii VS. Method of difference potentials and its applications. Berlin: Springer-Verlag; 2002.

[16] Utyuzhnikov SV. Generalized Calderón-Ryaben'kii potentials. IMA J Appl Math 2009;74(1):128-48.

[17] Breuer M, Kniazev B, Abel M. Development of wall models for LES separated flows using statical evaluations. Comput Fluids 2007;36(5):817-37.

[18] Vladimirov VS. Equations of mathematical physics. New York: Dekker; 1971. 